\title{
Review: mefloquine prevents malaria but has adverse effects that limit its acceptability in adults not immune to malarial infection
}

Croft AM, Garner P.Mefloquine for preventing malaria in non-immune adult travellers. Cochrane Database Syst Rev 2001;(1):CD000138 (latest version 28 Aug 2000).

QUESTION: In adult travellers not immune to malarial infection, is mefloquine more effective than placebo or other prophylaxis for preventing malarial episodes and adverse events?

\section{Data sources}

Published and unpublished studies were identified by searching Medline, EMBASE/Excerpta Medica, and LILACS from 1966 to July 2000; the Cochrane Infectious Diseases Group (to July 2000); and Science Citation Index; by handsearching 2 military medical journals $\{1945 \text { to } 1999\}^{*}$; and by handsearching for several subsequent issues the correspondence pages of journals yielding a published trial. Bibliographies of retrieved papers and standard textbooks of tropical medicine and conference proceedings were also searched. Personal contact was made with pharmaceutical companies, authors, and investigators.

\section{Study selection}

Randomised or quasirandomised controlled trials were selected if participants were adults not immune to malaria, were prescribed prophylactic drugs for $<12$ months, and were travelling to endemic malarious regions. Randomised tolerability studies were also included if they were done on pretravel or nontravelling volunteer participants. Studies were excluded if they examined a regimen no longer used for prophylaxis because of severe side effects.

\section{Data extraction}

Data were extracted on participant characteristics, methods, details of intervention or placebo, outcomes, and study quality. Main outcome measures were malarial illness and withdrawal for any reason. Secondary outcomes were adherence at first assessment and reported symptoms.

\section{Main results}

10 trials met the selection criteria. Mefloquine was better than placebo (table) but not other prophylaxis (1 case in 1 study) for preventing malarial episodes. Mefloquine was more likely than placebo (4 studies) (table) but not other prophylaxis (4 studies) to cause withdrawal from the study. Mefloquine was more likely than other prophylaxis to cause fatigue (4 studies) and insomnia (4 studies) (table). Non-adherence at first assessment (4 studies), depression (3 studies), dreams (4 studies), headache (6 studies), diarrhoea (6 studies), vomiting (4 studies), fever (2 studies), itch (3 studies), and non-malarial death or admission to hospital (2 studies) were also similar between mefloquine and other prophylaxis. Trial results for abdominal pain, anorexia, and nausea were heterogeneous.

\section{Conclusion}

In adult travellers not immune to malarial infection, mefloquine prevents malaria but has adverse effects that limits its acceptability.

*Information provided by author.

Mefloquine (Mef) v placebo (Pl) or other prophylaxis (Other) in adult travellers not immune to malarial infectiont

\begin{tabular}{lllll}
\hline Outcomes & Comparison & Event rates & RRR (95\% CI) & NNT (CI) \\
\hline Malaria (1 study) & Mef $v$ PI & $0 \% v 77 \%$ & $100 \%(93$ to 100$)$ & 2 (2 to 2$)$ \\
\hline & & $\begin{array}{l}\text { Weighted event } \\
\text { rates }\end{array}$ & RRI (CI) & NNH (CI) \\
Withdrawal & Mef $v$ PI & $4.3 \% v 1.2 \%$ & $238 \%(62$ to 603$)$ & $33(21$ to 72$)$ \\
\hline Fatigue & Mef $v$ Other & $7.9 \% v 5.3 \%$ & $50 \%(0$ to 125$)$ & $39(20$ to 1000$)$ \\
\hline Insomnia & Mef $v$ Other & $14.7 \% v 9.5 \%$ & $55 \%(15$ to 108$)$ & $20(12$ to 56$)$ \\
\hline
\end{tabular}

†A fixed effects model was used. Abbreviations defined in glossary; RRR, RRI, NNT, NNH, and Cl calculated from data in article.

\section{COMMENTARY}

Croft and Garner provide a sound systematic review of 10 randomised controlled trials on the chemoprophylactic effect of mefloquine in adults not immune to malaria. Analysis of the results confirms that mefloquine is better than placebo and its prophylactic effect is equivalent to that of other antimalarial drugs. The evaluated studies, however, are limited to a heterogeneous target population comprising military personnel and tourists, whose adherence to the treatment differs.

The authors showed that some side effects, such as fatigue and insomnia, occur more frequently in those receiving mefloquine than in those receiving other antimalarial drugs. The slow rate to achieve therapeutic blood concentrations, along with the long elimination half life (13 to 26 days), lead to long periods of suboptimal blood concentrations, which may favour the appearance of mefloquine resistant strains of the malaria parasite. This possibility as well as the safety, cost benefit ratio, and therapeutic effectiveness of mefloquine demand further investigation. The cost of a 2 week prophylactic course of the drug is approximately US $\$ 34$.

We need to keep in mind that mefloquine and most of the other drugs used for malaria chemoprophylaxis are blood schizonticides that have no effect on sporozoites and the pre-erythrocytic forms that infect liver cells. Therefore, they prevent neither infection by mosquitoes nor the possibility of later relapses by Plasmodium vivax or P ovale.

Until further information is available, it seems reasonable to prescribe mefloquine for malaria prophylaxis exclusively for people who travel to areas of chloroquine resistant malaria and who have no contraindications (eg, no present or previous symptoms of seizures or psychiatric illness or an underlying cardiac conduction disturbance). 